\title{
Erratum to: Preoperative predictive factors and further risk stratification of biochemical recurrence in clinically localized high-risk prostate cancer
}

\author{
Riu Hamada ${ }^{1}$ Jun Nakashima ${ }^{1} \cdot$ Makoto Ohori $^{1} \cdot$ Yoshio Ohno $^{1} \cdot$ Osamu Komori $^{2}$ • \\ Kunihiko Yoshioka $^{1} \cdot$ Masaaki Tachibana $^{1}$
}

Published online: 8 January 2016

(C) Japan Society of Clinical Oncology 2016

\section{Erratum to: Int J Clin Oncol \\ DOI 10.1007/s10147-015-0923-3}

Unfortunately, the name of the sixth author was incorrectly published in the original article. The corrected author's name (Kunihiko Yoshioka) is published with this erratum.

Also, in the abstract, introduction and footnotes of tables, "prostate serum antigen (PSA)" should read "prostate specific antigen (PSA)".

The online version of the original article can be found under doi:10.1007/s10147-015-0923-3.

Riu Hamada

hamao1103@yahoo.co.jp

1 Department of Urology, Tokyo Medical University Hospital, Tokyo Medical University, 6-7-2 Nishi-shinjyuku, Shinjuku-ku, Tokyo 160-8582, Japan

2 Department of Mathematical Analysis and Statistical Inference, The Institute of Statistical Mathematics, Tokyo, Japan 